Estudos Linguísticos

Linguistic Studies 



\title{
LE RÔLE DU MANUEL DANS LE PROCESSUS \\ D'ENSEIGNEMENT/APPRENTISSAGE DE FLE
}

\section{The role of the textbook in the process of teaching/learning French as a foreign language}

\author{
Christianne Benatti Rochebois*
}

\begin{abstract}
RESUMO
Em qual medida os métodos de FLE (Francês Língua Estrangeira) proporcionam os requisitos mínimos para encorajar as trocas entre as culturas respeitando a diversidade cultural? Esses métodos consideram a diversidade de contextos onde o francês é ensinado? Nós nos propomos nesta reflexão mostrar por quais meios os métodos tratam a diversidade cultural na era da globalização. O livro didático de base pode ser muito útil, à condição de não se limitar a uma abordagem puramente descritiva, etnográfica ou culturalista, e de levar o aprendiz a formular hipóteses sobre as razões que condicionam os hábitos ou comportamentos diferentes. o Quadro Europeu Comum de Referência para as línguas propõe aos professores trabalhar sobre diferentes aspectos dos traços distintos característicos de uma sociedade e de sua cultura. Nesta perspectiva, selecionamos uma lista de temas específicos para "abrir" o aprendiz à aquisição de conhecimentos socioculturais, a ter outros olhares da realidade e a se conscientizar da nãouniversalidade de sua cultura. Constituímos para isso uma tabela de análise para estudar o conjunto do conteúdo sócio-cultural dos manuais, cujos tópicos foram formuladas em função do nosso objetivo especifico, analisar os conteúdos sócio-culturais dos manuais sob o ângulo da diversidade.
\end{abstract}

Palavras-chave: Francês Lingua Estrangeira; métodos; diversidade cultural. 


\begin{abstract}
To what extent do methods of French as a Foreign Language (FFL) deploy the means required to encourage the exchanges among cultures while respecting cultural diversity? Do these methods take into account the array of contexts under which French is taught? In this paper we intend to show the means by which these methods choose to treat cultural diversity. The textbook can prove very useful as long as one pays attention not to limit oneself to a purely descriptive, ethnographic or culturalist approach, and makes sure to enable the learner to formulate hypotheses about the reasons which condition different habits or behaviors.

The Common European Reference Guidelines for Languages propose that teachers should work on different aspects of characteristic distinctive features of a society and its culture. From that perspective, we have compiled a list of themes that are appropriate to trigger the learner's acquisition of sociocultural knowledge, and to enable him/her to have other viewpoints on reality, as well as to be aware of the non-universal character of his/her own culture. We have thus arrived at an analytical framework to study the whole sociocultural content of textbooks, whose categories were set up specifically to serve our purpose: the analysis of sociocultural content of textbooks under the prism of diversity.
\end{abstract}

Keywords: French as a Foreign Language; methods; cultural diversity.

\title{
INTRODUCTION
}

Les débats des dernières décennies sur la culture dans la didactique de langues étrangères sont nés de la nécessité de former aujourd'hui des individus conscients et respectueux de la diversité culturelle, et mieux préparés à interagir socialement avec l'Autre. Cette diversité ne se réfère pas seulement à la pluralité des pays, mais surtout, à la diversité de l'espèce humaine. De ce fait, l'expression et l'interprétation de la culture doivent être vues comme des expressions et interprétations multiples, puisque dépendantes de la diversité des contextes, des situations, des expériences, des relations auxquels chacun, en tant qu'individu, est exposé quotidiennement. 
La complexité de la question suppose, de notre point de vue, un examen attentif des manuels de FLE de manière à y repérer comment la culture et la langue française y sont traitées, dès lors que langue et culture ne peuvent se réduire à l'acquisition de connaissances et au domaine de structures linguistiques, mais doivent être reconnues en tant que fait social, produit d'interaction entre les individus.

La sensibilisation à la diversité des points de vue et aux interprétations d'une même communauté culturelle représente, pour nous, le point de départ pour la compréhension de la manifestation de la culture à travers les individus, selon leur histoire personnelle, leur groupe social, leur profession, leur âge, leur région d'origine, leur expérience de vie. Nous sommes ainsi transmetteurs de culture. Comme l'affirme Pretceille (1996, p. 45), ce sont les individus porteurs d'une culture qui sont en relation. La tentative de formaliser une culture, en la généralisant à toute une société avec ses traits et ses comportements, fragmente la réalité et conduit à nier sa complexité et son hétérogénéité.

Les discussions sur le comment enseigner et apprendre une langue étrangère sont centrales pour les professeurs et chercheurs en didactique, quand ils réfléchissent, à partir des actions menées en salle de classe et aussi en dehors, aux approches qui orientent la pratique pédagogique. Le plus grand problème est la recherche de la cohérence et de l'équilibre de leurs actions, en vue d'établir un pont, un lien entre ce que les enseignants veulent idéalement et théoriquement faire, et ce qu'ils pratiquent (ou pensent pratiquer) quand ils enseignent. Dans la plupart des cas, nous avons une conscience claire de ce que nous ne voulons pas faire, mais nous ne savons pas toujours comment faire autrement. D'un autre côté, nous pensons connaître la bonne façon d'agir, mais nous faisons face à des situations dans lesquelles nos croyances sont mises à l'épreuve et nous montrent que parfois il n'est pas possible d'agir dans le sens que nous voulons. Les pratiques d'enseignement sont marquées par cette tension entre ce que nous planifions et ce que nous réussissons à pratiquer.

Dans une perspective dialogique, centrée sur les relations interculturelles, les expériences d'enseigner et d'apprendre s'inscrivent dans 
un aller/retour constant entre la théorie et la pratique, la construction et la déconstruction des signifiés, le vécu de l'apprenant et l'expérience de l'enseignant, le sujet des actions et le rôle de l'observateur. La salle de classe est le lieu privilégié, le milieu où ces rapports s'établissent, puisque c'est là où les conflits, les tensions et les écarts (dus à la rencontre de cultures différentes et du choc d'aspects sociaux, politiques, psychologiques, affectifs, etc.) peuvent être négociés pour l'ouverture d'un nouvel espace de construction d'une connaissance formée par les contributions de tous.

\section{LE MATÉRIEL-SOURCE}

Dans le panorama des recherches en didactique de langues étrangères, une autre question préoccupe les enseignants et chercheurs depuis de nombreuses années : celle du matériel de base utilisé en salle de classe, plus précisément dans notre cas, le manuel de FLE. Il est souvent critiqué par son inadaptation aux situations dans lesquelles des motivations et des besoins différents selon les apprenants sont en jeu, et où interfèrent des cultures variées présentes en salle de classe. D'une manière générale, on peut noter que les manuels n'offrent pas aux acteurs du processus d'enseignement/apprentissage beaucoup d'espace pour créer ; organisés de manière centralisée et uniforme, ils contiennent des indications qui dirigent l'action de l'enseignant à travers la présentation de contenus programmés, selon les étapes ou les unités distribuées dans un ordre prétabli. Les apprenants et les enseignants se voient " liés » aux instructions et aux contenus imposés par le manuel.

Dans une publication qui réunit des contributions de plusieurs chercheurs brésiliens (Coracini, 1999), la question de la fonction du livre didactique comme lieu de stabilisation dans le processus d'enseignement/apprentissage en salle de classe est examinée, sous divers éclairages. Le manuel indique ce qui doit être enseigné, comment cela doit être enseigné, et reflète une conception de ce qu'est apprendre. Apprendre, selon un point de vue largement diffusé en salle de classe, c'est appréhender les contenus offerts par le manuel, et la fonction de l'enseignant, qui a évidemment une autonomie de décision, est de transmettre ces contenus 
selon leur présentation dans le manuel.

Pour ce qui concerne l'enseignement/apprentissage spécifique du FLE, le constat n'est pas différent. Le besoin est manifeste de livres et de matériels en nombre qui abordent la dimension culturelle comme moyen d'intégration et de production d'un discours partagé ; mais les enseignants ont aussi besoin de matériels plus flexibles, qui ne modèlent pas de manière rigide les activités/contenus, et qui permettent à l'apprenant de voir avec ses propres yeux, d'apprendre avec son expérience et avec celles des autres partenaires engagés dans le processus d'enseignement/apprentissage (et avec lesquels s'établit un dialogue interculturel). Dans les manuels de FLE conçus en France jusqu'aux années 80, les contenus culturels véhiculés concernent presque toujours des aspects habituels de la culture française, exposés à travers des échantillons de langue hors contexte, c'est-à-dire, très généraux, éloignés des réalités francophones, ces éléments servant prioritairement à pratiquer les aspects formels de la langue.

A partir des années 80 l'approche communicative va conduire à des changements majeurs par rapport aux pratiques précédentes, en prenant appui, tout d'abord, sur le savoir communiquer en fonction de la situation et de l'intention de communication. En mettant en valeur, ensuite, les besoins langagiers définis en termes de fonctions langagières. Ces deux priorités vont suggérer le développement d'un matériel conçu pour des publics particuliers, comme par exemple, les jeunes adolescents, les étudiants du troisième cycle, ou les adultes intéressés au «français des affaires ». La préférence va alors être accordée à des documents authentiques plus liés aux réalités culturelles, où "les supports d'apprentissage seront élaborés de manière à ce qu'ils soient proches de l'authentique et des types d'échanges qui existent dans la réalité »(Cuq, 2005, p. 267).

Dans les années 1990, la traduction didactique des données des sciences de la communication va favoriser le recours aux éléments de la vie quotidienne au niveau 1 d'apprentissage de la langue étrangère, et aux textes plus complets, plus culturellement élargis au niveau avancé. Cela va provoquer un décalage important de l'enseignement de la culture du niveau 1 par rapport au niveau 2. Les méthodes prétendent s'adresser à de jeunes adultes qui ont des sujets d'intérêt plus larges et plus engagés dans les 
réalités socioculturelles que les simples éléments du quotidien.

Dans les années 2000, la publication du Cadre européen commun de référence pour l'apprentissage et l'enseignement des langues, "définit non seulement les niveaux de compétence qui permettent de mesurer les progrès de l'apprenant à chaque étape de l'apprentissage (...) mais offre également une base commune pour l'élaboration de programmes de langues vivantes et de référentiels " (Cuq, 2005, p. 269).

Il y a ainsi un danger de standardisation des manuels par rapport aux compétences générales que l'apprenant d'une langue étrangère doit acquérir, telles qu'elles sont désignées par le Cadre européen commun de références pour les langues. Le nœud du problème réside dans le traitement des choix culturels des auteurs des manuels.

Les manuels élaborés d'après l'approche communicative sont généralistes et négligent les particularités des publics concrets. Dû à cela, des enseignants de FLE au Brésil ont été tentés de chercher ailleurs ou de produire leur propre matériel, afin de disposer d'éléments novateurs, mieux adaptés au contexte local, de sorte à rendre leur tâche plus efficace. Cependant, comme l'observe Coracini (1999, p. 23), ils continuent à procéder sur le même mode, puisque [...] a sua organização, os princípios que a norteiam, a imagem de aluno que veiculam já estão incorporados no professor (note 1). Autant dire que le manque de matériel ne les rend pas plus créatifs : ils procèdent de manière assez dirigée, présentent les contenus dûment dosés, préétablissant ce qui doit être appris à partir de telle ou telle activité.

Alors que l'enseignant devrait avoir pour objectif de mettre l'apprenant, en tant que sujet critique et participatif, dans son processus d'enseignement/apprentissage, la réalité telle que nous la percevons est bien différente. Il y a une difficulté considérable à casser le modèle rigide d'actions promu par le manuel. En ce sens, ce n'est pas tant la présence du manuel qui doit être abolie, puisqu'il peut être un grand allié, mais son usage hégémonique, unique source de connaissance disponible dans une grande partie du territoire brésilien, où les ressources médias ne sont pas encore disponibles au grand public.

Le manuel peut constituer une source importante d'interaction et de soutien pour le développement des activités en salle de classe, et conduire à 
des résultats d'une plus grande qualité, à condition d'être flexible, c'est-àdire, de ne pas prédéterminer ce qui doit être dit ou fait pour l'apprentissage. Il pourrait présenter des scénarios variés pour planifier les unités et les activités selon différents niveaux de difficultés, laissant l'enseignant libre de les utiliser selon les besoins et les opportunités concrètes vécues dans la salle de classe.

Enseigner/apprendre suscite des échanges, des négociations, et conduit à la construction conjointe de signifiés. L'enseignant, en ce sens, doit considérer le manuel source comme support qui l'aidera à jouer pour et avec les apprenants, les incitant à penser, à réfléchir, à utiliser les stratégies et les expériences qu'ils apportent en classe, en plus de les stimuler à établir un dialogue entre leur langue/culture et la langue/culture cible.

L'élaboration de manuels, que ce soit dans la manière dont ils sont organisés, ou dans la sélection des contenus pour aborder la langue/culture, doit être accompagnée d'une réflexion sur l'impact des situations concrètes d'apprentissage et sur le comportement d'enseignants et d'apprenants en contact avec ces matériels.

Dans le travail de recherche présenté ici, nous prendrons comme point de départ le débat d'ensemble ouvert au Brésil sur la didactique des langues, particulièrement sur les questions liées aux rôles du manuel, de l'enseignant et de l'apprenant, en étudiant comment les méthodes (en l'occurrence, il s'agit des manuels retenus pour l'expérimentation : Connexions, Reflets et Francês) traitent la diversité culturelle et s'il y a ouverture à la diversité des contenus culturels permettant une approche plurielle (culture des régions, de la France d'Outre-Mer et de la francophonie).

L'insertion d'une composante culturelle dans les méthodes d'apprentissage de FLE est une constante dans les manuels de langue. Toutefois, l'approche proposée est souvent centrée sur la connaissance de caractéristiques culturelles françaises (comportement, habitudes...) qui, par ailleurs, recouvrent généralement les mêmes thèmes correspondant aux représentations françaises les plus répandues dans le monde : la gastronomie, la mode, la famille, les loisirs. La présentation des contenus culturels conduit souvent à survaloriser la culture cible, et la dimension interculturelle s'en trouve ainsi réduite à un questionnement de l'apprenant 
sur ses propres comportements et valeurs culturelles selon une démarche comparative.

D'après Holtzer (2003, p. 67), le « contact » entre les cultures source et cible, leur interdépendance, qui fonde le concept d'interculturel, n'apparaît que de façon elliptique dans les méthodes de langue en raison du caractère généraliste des manuels utilisables dans tous les pays et dont les publics visés ne sont pas culturellement identifiés. Il conviendrait d'adapter chaque méthode au pays - ou au groupe de pays proches sur le plan culturel quand c'est possible -, ce qui ne semble pas réalisable pour les éditeurs qui font prévaloir une logique économique.

Il s'agit naturellement d'adapter les méthodes au public - à l'âge et aux intérêts des apprenants, à leurs caractéristiques socioculturelles, et au niveau de connaissance langue/culture. Les matériaux proposés doivent être accessibles (c'est-à-dire, à la portée de l'apprenant tout seul) et les supports diversifiés ; les documents, autant que possible, authentiques : textes de différents types (journalistiques, littéraires et autres), images (dessins, reproductions ou photos), petits documents de la vie quotidienne (tickets, étiquettes, brochures, etc.), enregistrements (de fiction, de journaux télévisés, etc.), documents multimédia (cédéroms, sites internet...).

Un rapide parcours des méthodes de FLE les plus fréquemment utilisées, et même une analyse plus poussée, ne font que confirmer une image stéréotypée et très simplifiée de la France. Il faut pourtant reconnaître qu'un progrès certain a été accompli quant à l'introduction de la culture dans l'enseignement du FLE si on se reporte à la situation d'il y a une trentaine d'années, par exemple. Pour en avoir une image claire, il suffit de comparer un manuel classique de l'époque, par exemple, La France en Direct ${ }^{1}$, avec les méthodes actuelles de FLE. Celles-ci offrent des matériaux plus riches se rapportant à la culture française : différents textes, photos, reproductions, etc.

Malgré cela, les informations fournies souvent sous forme concise,

${ }^{1}$ La France en Direct, CAPELLE J. et G. et alii, Paris, Hachette,1969. 
des encadrés sommaires, des photos typiques, tous ces matériaux contribuent à une représentation partielle des réalités françaises. Nous percevons surtout une France moyenne, sans grands contrastes ou particularismes. La société française apparaît comme un ensemble harmonieux quasiment homogène, où les conflits, les clivages ou les disparités sont généralement absents. On souligne les traits traditionnels, on indique les lieux et les sites célèbres, l'atmosphère est plaisante et agréable. Les images de la diversité régionale dans l'Hexagone et aussi des pays francophones sont rares et la France de la mosaïque des cultures, du métissage culturel et social, la France des exclus et de la fracture sociale, est presque absente.

C'est toujours à partir de la réalité française représentée dans le manuel que l'apprenant est amené à comparer et à réfléchir sur sa propre réalité, et s'agissant d'un apprenant non européen, ou venant d'un pays « en voie de développement " par exemple, ce point de repère risque d'être compris comme un modèle, un idéal de culture et de civilisation. Quelle image de la France se dégage-t-elle des méthodes actuelles? S'agit-il d'une France ouverte à d'autres cultures?

L'espace représenté dans les manuels de FLE est, le plus souvent, celui de l'Hexagone. Il est vrai que, de plus en plus, on met en valeur autant la capitale que la province, mais qu'en est-il des pays francophones, qu'en est-il des pays où la langue française, même à petite échelle, est utilisée pour des raisons historiques, culturelles ou économiques?

De manière générale, selon Wolton, les informations sur l'étendue des rapports de la France avec les catégories de pays qui ont la langue française en commun, sont sans relief et même leurs citoyens sont ignorés ou réduits. De même, dans la presse française nous ne trouvons pas de rubriques régulières sur les pays francophones (sauf pour les conflits sociaux, les voyages officiels et quelques récits d'envoyés spéciaux hésitant entre dénonciation rapide et publi-reportage touristique (2003, P. 143)). Que dire des manuels de français langue étrangère conçus en France et utilisés partout dans le monde où l'on enseigne le français? Peut-être conviendrait-il de revoir les manuels de FLE dans le sens d'une vision moins nationale, plus 
ouverte à la diversité culturelle.

Comme toutes les langues, le français est en continuel changement. Des mots vieillissent et disparaissent ; des néologismes deviennent des mots courants et bien acceptés ; on se sert de mots empruntés à des langues étrangères ; les manières de prononcer se transforment peu à peu ; certaines constructions de phrase sont abandonnées, de nouvelles formulations s'imposent. Si le français change au cours du temps, il varie aussi dans l'espace. On ne parle pas exactement le même français en tous les points de la francophonie. C'est vrai aussi pour la France : même si la radio et la télévision tendent à imposer un modèle de français standard, les accents et différences linguistiques régionaux sont encore bien vivants.

Chaque pays ou chaque région francophone a développé des particularités qui font l'originalité du français qu'on y parle, à travers des conditions historiques, la situation géographique, et surtout à partir des contacts de langue. Ces variétés du français sont-elles évoquées dans les manuels de FLE?

Pour travailler sur l'intégration de ces cultures en classe il faut puiser sans modération du côté des contenus des chansons, des journaux, des films, des publicités, des guides de voyage, des photos, des sites internet, etc., avec le manuel de FLE comme support de base. L'apprenant brésilien de l'ère mondialisée a besoin de travailler dans un contexte d'ouverture à la diversité et sur un matériel qui prend en compte cette nécessité (il faut nous rappeler que le Brésil est lui-même un espace de diversité). En accordant à l'interculturel la place qu'il doit avoir dans les cours, nous pouvons espérer relancer la motivation, renouveler les manières d'apprendre et d'enseigner et surtout faire vivre la langue.

\section{LA GRILLE D’ANALYSE}

D'après Abdallah-Pretceille (2005), le discours culturel est un fort véhicule de messages dans un processus de communication, qui, selon le terrain, choisit ses arguments, ses formulations et ses implicites. Pour 
comprendre l'étendue d'une culture nous ne pouvons pas rester de simples spectateurs, il nous faut être attentifs. Il faut essayer de dépasser le premier degré et approfondir une analyse de la signification.

\begin{abstract}
De même 'qu'il est impossible d'interpréter un acte de communication dans les limites d'une analyse purement linguistique' (Bourdieu, Réponses, Paris, Seuil, 1992 : 118), il est impossible de comprendre une culture à partir d'une analyse purement culturaliste, c'est-à-dire, à partir de la connaissance de faits culturels. En tant que système symbolique, la culture n'est pas simplement un instrument au service du message mais aussi un instrument potentiel de domination. (Abdallah-Pretceille, 2005, p. 130)
\end{abstract}

La compréhension culturelle exige donc une perception des contextes, des objectifs, des individus et aussi des implicites et des non-dits. Elle doit prendre en compte le décalage existant entre la base de la production et celle de l'interprétation, tout comme la relation entre les deux.

Dans le cas spécifique du manuel de FLE, les faits culturels présentés par les auteurs sont révélateurs de leurs représentations d'une réalité culturelle chargée d'une intentionnalité spécifique (Abdallah-Pretceille, 2005, p. 133). Bien que ces auteurs prennent en considération le public visé, en relation avec l'âge et le niveau de connaissance de la langue, le choix des contenus culturels d'un manuel témoigne d'une universalisation des points de vue, en méconnaissant ou en ignorant les identités culturelles qui seront de l'autre côté de la relation de communication. Cette donnée peut entraîner des conflits et des malentendus culturels, qui peuvent être réduits grâce aux connaissances antérieures que les apprenants ont de la culture française, et à l'intervention de l'enseignant dans un réel accompagnement du dialogue interculturel en salle de classe.

Les sociétés contemporaines réclament un respect mieux assuré ainsi qu'une meilleure promotion de la diversité culturelle. Les institutions tiennent en général un discours sur une diversité culturelle qui doit devenir facteur de dialogues et de valeurs partagées, et exprimer l'harmonie des éléments dans une totalité non homogène. Mais la diversité n'est pas 
nécessairement harmonie et l'actualité nous le montre tous les jours. La diversité sociale, raciale, culturelle est aussi conflictuelle.

Dans cette perspective, nous voulons vérifier dans notre travail si le manuel de FLE, en tant que bien culturel (ainsi que les livres, journaux, films, produits multimédias, etc.), est à la fois le reflet et l'expression d'identités particulières, celles de la France dans la diversité de ses composants (régionales, ethniques...) et celles du monde francophone.

Nous avons donc constitué une grille d'analyse pour étudier l'ensemble du contenu socioculturel des trois manuels choisis pour notre recherche, Connexions, Reflets et Francês. Pour le choix des rubriques de la grille, nous nous sommes basé sur les travaux de Maria Cecilia Bertoletti et Patrick Dahlet - Manuels et matériels scolaires pour l'apprentissage du FLE, 1984 -, de Anne Marie Thierry - Analyse de méthodes français langue étrangère, 1987 et réactualisé en 1991 -, et sur la recherche de Nathalie Auger - Les représentations interculturelles dans les manuels de français langue étrangère en usage dans l'Union européenne, 2003. Une sélection et des modifications des rubriques ont été faites en fonction de notre objectif spécifique, l'analyse des contenus socioculturels des manuels sous l'angle de la diversité.

Nous distinguerons trois catégories de contenus : le contenu iconographique, textuel et socioculturel. Chaque contenu peut se décrire à partir des indicateurs présentés dans les tableaux suivants :

\begin{tabular}{|c|l|l|}
\hline \multicolumn{3}{|c|}{ CONTENU ICONOGRAPHIQUE } \\
\hline TYPOLOGIE & \multicolumn{1}{|c|}{ ORIGINE } & \multicolumn{1}{|c|}{ FONCTION } \\
\hline a) photos & Documents : & a)culturelle \\
b) images & b)ethnographique \\
publicitaires & a)authentiques & c)sémantique \\
c) bandes dessinées & b)remaniés & d)situationnelle \\
d)reproduction de & c)fabriqués & e)illustrative \\
documents officiels & & \\
e) dessins & & \\
f) cartes, plans, & & \\
calendriers & & \\
g)autres & & \\
\hline
\end{tabular}




\begin{tabular}{|c|c|c|}
\hline \multicolumn{3}{|c|}{ CONTENU TEXTUEL } \\
\hline TYPOLOGIE & ORIGINE & FONCTION \\
\hline $\begin{array}{l}\text { a) dialogues } \\
\text { conversationnels } \\
\text { b) textes littéraires } \\
\text { c) e-mails } \\
\text { d) chansons } \\
\text { e) articles de presse } \\
\text { f) messages } \\
\text { publicitaires } \\
\text { g) autres (phrases, } \\
\text { bulles, mots croisés, } \\
\text { devinettes) }\end{array}$ & $\begin{array}{l}\text { Documents : } \\
\text { a) authentiques } \\
\text { b) remaniés } \\
\text { c) fabriqués }\end{array}$ & $\begin{array}{l}\text { a) culturelle } \\
\text { b) ethnographique } \\
\text { c) sémantique } \\
\text { d) situationnelle } \\
\text { e) illustrative }\end{array}$ \\
\hline
\end{tabular}

\section{CONTENU SOCIO-CULTUREL}

a) Présentation du thème (d'intérêt général, centré sur l'Hexagone, sur une région, sur un pays francophone)

b) Caractérisation socioculturelle du contexte et des personnages (nationalité, âge, profession, milieu social)

c) Caractères des rapports sociaux (familiaux/professionnels, détendus/conflictuels, neutres)

La question n'est pas ici de débattre sur le point de savoir si chacun des manuels analysés, dans son aire culturelle d'origine, appelle une méthodologie spécifique de diversité culturelle, mais de savoir si les textes choisis pour transmettre cette diversité dans un contexte donné à des fins très particulières, peuvent s'adapter au public d'apprenants brésiliens et servir ainsi de carrefour dans un espace où circule l’hétérogénéité. 


\section{REFERÊNCIAS}

ABDALLAH-PRETCEILLE, M. Vers une pédagogie interculturelle. Paris: Anthropos, 1996.

. PORCHER, L. Education et communication interculturelle. Paris: Presses Universitaires de France, 2005.

CAPELLE, G., GIDON, N. Reflets. Paris: Hachette, 1999.

CORACINI, M.J. O livro didático nos discursos da Lingüística Aplicada e da sala de aula. In: 1999.

(org). Interpretação, Autoria e Legitimação do Livro Didático. Campinas: Pontes, p. 17-26,

CUQ, J-P., GRUCA, I., Cours de didactique du français langue étrangère et seconde. Grenoble: PUG, 2005.

FONTOURA de PAULA, E. Francês, livro 1. Belo Horizonte: Editora Universidade, 1997.

HOLTZER,G. Enseigner les cultures à l'ère de la mondialisation. Bulletin de l'université, Lomossov n 1, série 9, 2003.

MÉRIEUX, R., LOISEAU, Y. Connexions. Paris: Les Éditions Didier, 2004.

WOLTON, D. L'autre mondialisation. Paris: Editions Champs Flammarion, 2003.

Submetido em: 13/03/2011

Aceito em: 29/06/2011 